\title{
Pricing Volatility Derivatives under the Modified Constant Elasticity of Variance Model
}

\author{
Leunglung Chan * and Eckhard Platen ${ }^{\dagger}$
}

June 4, 2015

\begin{abstract}
This paper studies volatility derivatives such as variance and volatility swaps, options on variance in the modified constant elasticity of variance model using the benchmark approach. The analytical expressions of pricing formulas for variance swaps are presented. In addition, the numerical solutions for variance swaps, volatility swaps and options on variance are demonstrated.
\end{abstract}

Keywords: CEV model; volatility derivatives; benchmark approach.

\section{Introduction}

This paper considers the modified constant elasticity of variance (MCEV) model, which is an extension to the Black-Scholes-Merton model and the stylized minimal market model;

*School of Mathematics and Statistics, University of New South Wales, NSW, Australia; Email: Leung.chan@unsw.edu.au

${ }^{\dagger}$ Finance Discipline Group and School of Mathematical and Physical Sciences, University of Technology Sydney, PO Box 123, Broadway, NSW 2007, Australia; Email: Eckhard.Platen@uts.edu.au 
see [20]. The standard CEV model was originally introduced by [9]. The main advantages of using the CEV model are that it can account for the implied volatility smile and smirk by capturing the leverage effect.

The pricing of different kinds of options under the constant elasticity of variance (CEV) model have provided interesting and challenging research topics; see e.g. [2][11] [18][13][20]. The latter paper modeled the growth optimal portfolio (GOP) under the real world probability measure, where it is referred to as the MCEV model. The current paper will study volatility derivatives under this model.

Since the S\&P 500 volatility index VIX was introduced in 1993, there have been more and more volatility derivatives tradable on the exchanges or over the counter. The VIX index can be theoretically interpreted as the standardized risk-neutral expected realized variance; see [4][6]. Recent literature discussing volatility derivatives include [12][5][7][17][8].

We will apply the benchmark approach, documented in [20], which uses the GOP as the numéraire so that the contingent claims will be priced under the real world probability measure. This avoids the restrictive assumption on the existence of an equivalent risk neutral probability measure. As argued in [20], this measure seems not to exist for realistic models and does not exist for the MCEV model. In the following, we derive closedform formulas for variance swaps under the MCEV model and show numerical results for volatility derivatives.

\section{Volatility Derivatives}

A variance swap is a forward contract on annualized variance. Let $\sigma_{0, T}^{2}$ denote the realized annualized variance of the log-returns of a diversified equity index or related futures over the life of the contract such that

$$
\sigma_{0, T}^{2}:=\frac{1}{T} \int_{0}^{T} \sigma_{u}^{2} d u
$$


Assume that one can trade the underlying futures or index price at discrete times $t_{i}=i \Delta$ for $i \in\{0,1, \ldots\}$ with time step size $\Delta>0$. The period $\Delta$ between two successive potential trading times is typically the length of one day. $S_{t_{i}}^{\delta_{*}}$ denotes the index price at time $t_{i}$ for $i \in\{0,1,2, \ldots\}$.

Let $\left(\Omega, \mathcal{A}_{T}, \underline{\mathcal{A}}, \mathcal{P}\right)$ denote the underlying filtered probability space satisfying usual conditions. Here $\mathcal{P}$ is the real world probability measure and $\underline{\mathcal{A}}=\left(\mathcal{A}_{t}\right)_{t \in[0, T]}$ the respective filtration. For simplicity, assume throughout the paper that the interest rate $r>0$ is constant. Furthermore, we assume that the index is the GOP $S_{t}^{\delta_{*}}$, also called benchmark of the market. We call any price or payoff denominated in units of the GOP the respective benchmarked price. We employ in this paper the real world pricing formula, which provides for a replicable $\mathcal{A}_{\bar{T}}$-measurable contingent claim $H_{\bar{T}}$ with $E\left(\frac{\left|H_{\bar{T}}\right|}{S_{\bar{T}}^{\delta_{*}}}\right)<\infty$ the real world pricing formula

$$
V_{t}=S_{t}^{\delta_{*}} E\left[\frac{H_{\bar{T}}}{S_{\bar{T}}^{\delta_{*}}} \mid \mathcal{A}_{t}\right]
$$

for all $t \in[0, \bar{T}], \bar{T} \in[0, T]$; see $[20]$.

Let $K_{v}$ denote the delivery price for realized variance and $L$ the notional amount of the swap in dollars per annualized variance point. Then, the payoff of the variance swap at expiration time $T$ is given by $L\left(\sigma_{0, T}^{2}-K_{v}\right)$.

A volatility swap is a forward contract on annualized volatility. Let $K_{s}$ denote the annualized volatility delivery price and $L$ the notional amount of the swap in dollar per annualized volatility point. Then, the payoff function of the volatility swap is given by $L\left(\sigma_{0, T}-K_{s}\right)$, where $\sigma_{0, T}=\sqrt{\sigma_{0, T}^{2}}$.

Additionally, we will consider the payoffs of call options on variance, defined by $\left(\sigma_{0, T}^{2}-\right.$ $K)^{+}$, as well as, the payoffs of put options on variance, defined by $\left(K-\sigma_{0, T}^{2}\right)^{+}$, where $a^{+}=\max (0, a)$. 


\section{Modified Constant Elasticity of Variance Model}

As shown in [14], the MCEV model for the GOP is obtained when the volatility of the GOP takes the form

$$
\left|\theta_{t}\right|=\left(S_{t}^{\delta_{*}}\right)^{a-1} \psi
$$

for $t \in[0, \infty)$ with exponent $a \in(-\infty, \infty), a \neq 1$, and scaling parameter $\psi>0$. From [14], recall that the discounted GOP satisfies the SDE

$$
d S_{t}^{\delta_{*}}=\left(r S_{t}^{\delta_{*}}+\left(S_{t}^{\delta_{*}}\right)^{2 a-1} \psi^{2}\right) d t+\left(S_{t}^{\delta_{*}}\right)^{a} \psi d W(t)
$$

for $t \in[0, T]$. Now set $X_{t}=\left(S_{t}^{\delta_{*}}\right)^{2(1-a)}$. Then we have

$$
d X_{t}=k\left(\vartheta-X_{t}\right) d t+\sigma \sqrt{X_{t}} d W(t)
$$

where $k=-2(1-a) r, \vartheta=-\frac{\psi^{2}(3-2 a)}{2 r}, \sigma=2 \psi(1-a)$. Note that $X_{t}$ is a space-time changed squared Bessel process of dimension $\delta=\frac{3-2 a}{1-a}$; see [15].

\section{Explicit Formula for Variance Swaps}

Due to (2.2) the value of a variance swap $V_{v}\left(t, S_{t}^{\delta_{*}}\right)$ at time $t=0$ is given by:

$$
V_{v}\left(0, S_{0}^{\delta_{*}}\right)=S_{0}^{\delta_{*}} E\left[\frac{L\left(\sigma_{0, T}^{2}-K_{v}\right)}{S_{T}^{\delta_{*}}}\right]=S_{0}^{\delta_{*}} L E\left[\frac{\sigma_{0, T}^{2}}{S_{T}^{\delta_{*}}}\right]-S_{0}^{\delta_{*}} L K_{v} E\left[\frac{1}{S_{T}^{\delta_{*}}}\right] .
$$

Hence, the evaluation of the price of a variance swap can be reduced to the problem of calculating the expected value $E\left[\frac{\sigma_{0, T}^{2}}{S_{T}^{\delta_{*}}}\right]$ of the benchmarked realized annualized variance and the zero coupon bond $B_{T}\left(0, S_{0}^{\delta_{*}^{*}}\right)=S_{0}^{\delta_{*}} E\left[\frac{1}{S_{T}^{\delta_{*}^{*}}}\right]$.

As follows from [19], the price of a zero-coupon bond $B_{T}\left(t, S_{t}^{\delta_{*}}\right)$, calculated at time $t$ with maturity $T$ under the given MCEV model, equals

$$
B_{T}\left(t, S_{t}^{\delta_{*}}\right)=e^{-r(T-t)} \chi^{2}\left(\Upsilon_{T} ; \frac{1}{1-a}\right)
$$


where

$$
\Upsilon_{T}=\frac{2 r}{\left|\theta_{t}\right|^{2}(1-a)[1-\exp \{-2(1-a) r(T-t)\}]}
$$

for $t \in[0, T]$ and $\chi^{2}(u, \nu)=1-\frac{\Gamma\left(\frac{u}{2} ; \frac{\nu}{2}\right)}{\Gamma\left(\frac{\nu}{2}\right)}$ for $u \geq 0$ and where $\Gamma(\alpha)$ for $\alpha>-1$ is the gamma function, and $\Gamma(.,$.$) is the incomplete gamma function; see [20].$

Furthermore, we have

$$
E\left[\frac{\sigma_{0, T}^{2}}{S_{T}^{\delta_{*}}}\right]=\frac{\psi^{2}}{T} E\left[\frac{\int_{0}^{T}\left(S_{s}^{\delta_{*}}\right)^{2(a-1)} d s}{S_{T}^{\delta_{*}}}\right]=\frac{\psi^{2}}{T} E\left[\frac{\int_{0}^{T} \frac{1}{X_{s}} d s}{X_{T}^{\frac{1}{2(1-a)}}}\right] .
$$

Lemma 4.1 Let $X=\left\{X_{t}: t \in[0, T]\right\}$ satisfy the $\operatorname{SDE}(3.3)$ and set $\beta=1+m-$ $\frac{1}{2(1-a)}+\nu / 2, m=\frac{1}{2}\left(\frac{2 k \vartheta}{\sigma^{2}}-1\right), \nu=\frac{2}{\sigma^{2}} \sqrt{\left(k \vartheta-\frac{\sigma^{2}}{2}\right)^{2}+2 \mu \sigma^{2}}, \mu>0$ and $X_{0}=x>0$. Then if $m>\frac{1}{2(1-a)}-\frac{\nu}{2}-1$, we have

$$
\begin{gathered}
E\left[\frac{\int_{0}^{T} \frac{d s}{X_{s}}}{X_{T}^{\frac{1}{2(1-a)}}}\right]=-\frac{d}{d \mu} \frac{1}{2^{\nu} x^{m}} e^{-\frac{2 k x}{\sigma^{2}\left(e^{k T}-1\right)}+k m t}\left(\frac{2 k e^{k T}}{\left(e^{k T}-1\right) \sigma^{2}}\right)^{-m+\frac{1}{2(1-a)}-\frac{\nu}{2}} \\
\times\left.\quad\left(\frac{4 k^{2} x}{\sigma^{4} \sinh ^{2}\left(\frac{k T}{2}\right)}\right)^{\nu / 2} \frac{\Gamma\left(1+m-\frac{1}{2(1-a)}+\frac{\nu}{2}\right)}{\Gamma(1+\nu)}{ }_{1} F_{1}\left(\beta, 1+\nu, \frac{2 k x}{\sigma^{2}\left(e^{k T}-1\right)}\right)\right|_{\mu=0} .
\end{gathered}
$$

Here the function ${ }_{1} F_{1}(., .,$.$) is the confluent hypergeometric function; see [8].$

Proof: Similar to Proposition 8.1 in [8], we can prove this. However, we provide an alternative proof as below.

For $\lambda>0$ and $\mu>0$, use the Corollary 5.9 of [10], the joint Laplace transform of $X_{T}$ 
and $\int_{0}^{T} \frac{d s}{X_{s}}$ admits the expression

$$
\begin{aligned}
& E\left(e^{-\lambda X_{T}-\mu \int_{0}^{T} \frac{d s}{X_{s}}}\right) \\
= & \frac{\Gamma(m+\nu / 2+1)}{\Gamma(\nu+1)} \frac{k \sqrt{x}}{\sigma^{2} \sinh (k T / 2)} x^{-\left(m+\frac{1}{2}\right)} \\
\times & \exp \left(\frac{k}{\sigma^{2}}\left(k \vartheta T+x-\frac{x}{\tanh (k T / 2)}\right)\right) \\
\times & \frac{\sigma^{2} \sinh (k T / 2)}{k \sqrt{x} \hat{\alpha}^{\left(m+\frac{1}{2}\right)}} e^{\frac{k^{2} x}{2 \sigma^{4} \sinh ^{2}(k T / 2) \hat{\alpha}}} M_{-\left(m+\frac{1}{2}\right), \nu / 2}\left(\frac{k^{2} x}{\sigma^{4} \sinh ^{2}(k T / 2) \hat{\alpha}}\right) .
\end{aligned}
$$

Here $\hat{\alpha}=\frac{k}{\sigma^{2}}\left(1+\operatorname{coth}\left(\frac{k T}{2}\right)\right)+\lambda$ and $M_{s, r}(z)$ is the Whittaker functions of the first kind.

Multiplying both sides of (4.6) by $\frac{\lambda^{\frac{1}{2(1-a)}-1}}{\Gamma\left(\frac{1}{2(1-a)}\right)}$ and integrating with respect to $\lambda$, gives

$$
\begin{aligned}
& \int_{0}^{\infty} E\left(e^{-\mu \int_{0}^{T} \frac{d s}{X_{s}}} e^{-\lambda X_{T}}\right) \lambda^{\frac{1}{2(1-a)}-1} \frac{d \lambda}{\Gamma\left(\frac{1}{2(1-a)}\right)}=\int_{0}^{\infty} \frac{\Gamma(m+\nu / 2+1)}{\Gamma(\nu+1)} \frac{k \sqrt{x}}{\sigma^{2} \sinh (k T / 2)} x^{-\left(m+\frac{1}{2}\right)} \\
& \times \exp \left(\frac{k}{\sigma^{2}}\left(k \vartheta T+x-\frac{x}{\tanh (k T / 2)}\right)\right) \frac{\sigma^{2} \sinh (k T / 2)}{k \sqrt{x} \hat{\alpha}^{\left(m+\frac{1}{2}\right)}} e^{\frac{k^{2} x}{2 \sigma^{4} \sinh ^{2}(k T / 2) \hat{\alpha}}} \\
& \times \quad M_{-\left(m+\frac{1}{2}\right), \nu / 2}\left(\frac{k^{2} x}{\sigma^{4} \sinh ^{2}(k T / 2) \hat{\alpha}}\right) \lambda^{\frac{1}{2(1-a)}-1} \frac{d \lambda}{\Gamma\left(\frac{1}{2(1-a)}\right)} .
\end{aligned}
$$

On the left-hand side, changing the order of integration gives the expression

$$
E\left(\exp \left(-\mu \int_{0}^{T} \frac{d s}{X_{s}}\right) X_{T}^{-\frac{1}{2(1-a)}}\right) .
$$

The right-hand side of (4.7) can be integrated explicitly in form of

$$
\begin{aligned}
& \frac{1}{2^{\nu} x^{m}} e^{-\frac{2 k x}{\sigma^{2}\left(e^{k t}-1\right)}+k m T}\left(\frac{2 k e^{k T}}{\left(e^{k T}-1\right) \sigma^{2}}\right)^{-m+\frac{1}{2(1-a)}-\frac{\nu}{2}} \\
\times & \left(\frac{4 k^{2} x}{\sigma^{4} \sinh ^{2}\left(\frac{k T}{2}\right)}\right)^{\nu / 2} \frac{\Gamma\left(1+m-\frac{1}{2(1-a)}+\frac{\nu}{2}\right)}{\Gamma(1+\nu)}{ }_{1} F_{1}\left(\beta, 1+\nu, \frac{2 k x}{\sigma^{2}\left(e^{k T}-1\right)}\right) .
\end{aligned}
$$


Differentiating the expression (4.8) and the expression (4.9) with respect to $\mu$ gives

$$
\begin{gathered}
E\left[\frac{\int_{0}^{T} \frac{d s}{X_{s}}}{X_{T}^{\frac{1}{2(1-a)}}}\right]=-\frac{d}{d \mu} \frac{1}{2^{\nu} x^{m}} e^{-\frac{2 k x}{\sigma^{2}\left(e^{k T}-1\right)}+k m T}\left(\frac{2 k e^{k T}}{\left(e^{k T}-1\right) \sigma^{2}}\right)^{-m+\frac{1}{2(1-a)}-\frac{\nu}{2}} \\
\times\left.\quad\left(\frac{4 k^{2} x}{\sigma^{4} \sinh ^{2}\left(\frac{k T}{2}\right)}\right)^{\nu / 2} \frac{\Gamma\left(1+m-\frac{1}{2(1-a)}+\frac{\nu}{2}\right)}{\Gamma(1+\nu)}{ }_{1} F_{1}\left(\beta, 1+\nu, \frac{2 k x}{\sigma^{2}\left(e^{k T}-1\right)}\right)\right|_{\mu=0} .
\end{gathered}
$$

Next we verify the integrability condition, that is if $m>\frac{1}{2(1-a)}-\frac{\nu}{2}-1$, then the integral of right-hand side of $(4.7)$ is finite. In (4.7), make the substitution $\xi=\left(\frac{k}{\sigma^{2}}(1+\right.$ $\left.\left.\operatorname{coth}\left(\frac{k T}{2}\right)\right)+\lambda\right)^{-1}$ to rewrite the expression of the right-hand side as

$$
\begin{aligned}
& \int_{0}^{\infty} \frac{\Gamma(m+\nu / 2+1)}{\Gamma\left(\frac{1}{2(1-a)}\right) \Gamma(\nu+1)} \frac{k \sqrt{x}}{\sigma^{2} \sinh (k T / 2)} x^{-\left(m+\frac{1}{2}\right)} \\
\times & \exp \left(\frac{k}{\sigma^{2}}\left(k \vartheta T+x-\frac{x}{\tanh (k T / 2)}\right)\right) \\
\times & \frac{\sigma^{2} \sinh (k T / 2)}{k \sqrt{x}} \xi^{\left(m+\frac{1}{2}\right)} e^{\frac{k^{2} x \xi}{2 \sigma^{4} \sinh ^{2}(k T / 2)}} \\
\times & M_{-\left(m+\frac{1}{2}\right), \nu / 2}\left(\frac{k^{2} x \xi}{\sigma^{4} \sinh ^{2}(k T / 2)}\right)\left(\frac{1}{\xi}-\frac{k}{\sigma^{2}}(1+\operatorname{coth}(k T / 2))\right)^{\frac{1}{2(1-a)}-1}\left(-\xi^{-2}\right) d \xi
\end{aligned}
$$

For small $\xi$, the integrand is proportion to

$$
\begin{aligned}
& \xi^{m-\frac{1}{2(1-a)}-\frac{1}{2}} e^{\frac{k^{2} x \xi}{2 \sigma^{4} \sinh ^{2}(k T / 2)}} M_{-\left(m+\frac{1}{2}\right), \nu / 2}\left(\frac{k^{2} x \xi}{\sigma^{4} \sinh ^{2}(k T / 2)}\right) \\
= & \xi^{m-\frac{1}{2(1-a)}+\frac{\nu}{2}}\left(\frac{k^{2} x}{\sigma^{4} \sinh ^{2}(k T / 2)}\right)^{\frac{1}{2}+\frac{\nu}{2}} F_{1}\left(1+m+\nu / 2,1+\nu, \frac{k^{2} x \xi}{\sigma^{4} \sinh ^{2}(k T / 2)}\right) \\
= & \xi^{m-\frac{1}{2(1-a)}+\frac{\nu}{2}}\left(\frac{k^{2} x}{\sigma^{4} \sinh ^{2}(k T / 2)}\right)^{\frac{1}{2}+\frac{\nu}{2}} .
\end{aligned}
$$

The above expression follows from the fact that $|\xi| \rightarrow 0,{ }_{1} F_{1}(a, b, 0)=1, b \neq-n$. This shows why we need $m>\frac{1}{2(1-a)}-\frac{\nu}{2}-1$. 
Now, we give an example for variance swaps. The values for the parameters of the model are set to $k=0.052, a=\frac{2}{3}, \vartheta=24.0385, \psi=1.5, m=\frac{3}{4}, \sigma=0.3162, x=1$, $L=1$ million dollars and $K_{v}=1$.

Table 4.1 displays the prices of variance swaps for various maturities.

Table 4.1: Prices of variance swaps
\begin{tabular}{|c|c|}
\hline maturities & Prices of variance swaps \\
\hline $1 / 6$ & 1.58587 \\
\hline 0.25 & 1.85638 \\
\hline 0.5 & 2.51737 \\
\hline 1 & 1.83401 \\
\hline 1.5 & 0.89961 \\
\hline 2 & 0.301646 \\
\hline
\end{tabular}

\section{Options on Variance}

According to (2.2), the value of a call option on variance at time zero is given by:

$$
C_{v}\left(0, S_{0}^{\delta_{*}}\right)=S_{0}^{\delta_{*}} E\left[\frac{\left(\sigma_{0, T}^{2}-K\right)^{+}}{S_{T}^{\delta_{*}}}\right]=S_{0}^{\delta_{*}} E\left[\left(\frac{\sigma_{0, T}^{2}}{S_{T}^{\delta_{*}}}-\frac{K}{S_{T}^{\delta_{*}}}\right)^{+}\right]
$$

Whereas the value of a put option on variance at time zero can be written as:

$$
\begin{aligned}
& P_{v}\left(0, S_{0}^{\delta_{*}}\right)=S_{0}^{\delta_{*}} E\left[\frac{\left(K-\frac{\psi^{2}}{T} \int_{0}^{T}\left(S_{s}^{\delta_{*}}\right)^{2(a-1)} d s\right)^{+}}{S_{T}^{\delta_{*}}}\right] \\
& =S_{0}^{\delta_{*}} E\left[\left(\frac{K}{X_{T}^{\frac{1}{2(1-a)}}}-\frac{\frac{\psi^{2}}{T} \int_{0}^{T} \frac{d s}{X_{s}}}{X_{T}^{\frac{1}{2(1-a)}}}\right)^{+}\right] \text {. }
\end{aligned}
$$


Let $h(K)=\left(\frac{K}{X_{T}^{\frac{1}{2(1-a)}}}-\frac{\frac{\psi^{2}}{T} \int_{0}^{T} \frac{d s}{X_{s}}}{X_{T}^{\frac{1}{2(1-a)}}}\right)^{+}$and $c=\frac{\psi^{2}}{T} \int_{0}^{T} \frac{d s}{X_{s}}$, then the Laplace transform

$$
\begin{aligned}
& \mathcal{L}(h(K))=\int_{0}^{\infty} e^{-z K}\left(\frac{K}{X_{T}^{\frac{1}{2(1-a)}}}-\frac{\frac{\psi^{2}}{T} \int_{0}^{T} \frac{d s}{X_{s}}}{X_{T}^{\frac{1}{2(1-a)}}}\right)^{+} d K \\
& =\int_{c}^{\infty} e^{-z K}\left(\frac{K}{X_{T}^{\frac{1}{2(1-a)}}}-\frac{c}{X_{T}^{\frac{1}{2(1-a)}}}\right) d K \\
& =-\left.\frac{K}{X_{T}^{\frac{1}{2(1-a)}} z} e^{-z K}\right|_{c} ^{\infty}-\left.\frac{1}{X_{T}^{\frac{1}{2(1-a)}} z^{2}} e^{-z K}\right|_{c} ^{\infty}+\left.\frac{c}{X_{T}^{\frac{1}{2(1-a)}} z} e^{-z K}\right|_{c} ^{\infty} \\
& =\frac{1}{X_{T}^{\frac{1}{2(1-a)}} z^{2}} e^{-z c}=\frac{1}{X_{T}^{\frac{1}{2(1-a)}} z^{2}} e^{\frac{-z \psi^{2}}{T} \int_{0}^{T} \frac{d s}{X_{s}}} .
\end{aligned}
$$

Inverting the Laplace transform gives

$$
\mathcal{L}^{-1}(h(K))=\frac{1}{2 \pi i} \int_{d-\infty i}^{d+\infty i} \frac{1}{X_{T}^{\frac{1}{2(1-a)}} z^{2}} e^{\frac{-z \psi^{2}}{T} \int_{0}^{T} \frac{d s}{X_{s}}} e^{z K} d z
$$

Hence,

$$
E\left[\left(\frac{K}{X_{T}^{\frac{1}{2(1-a)}}}-\frac{\frac{\psi^{2}}{T} \int_{0}^{T} \frac{d s}{X_{s}}}{X_{T}^{\frac{1}{2(1-a)}}}\right)^{+}\right]=\frac{1}{2 \pi i} \int_{d-\infty i}^{d+\infty i} \frac{e^{z K}}{z^{2}} E\left[\frac{e^{\frac{-z \psi^{2}}{T} \int_{0}^{T} \frac{d s}{X_{s}}}}{X_{T}^{\frac{1}{2(1-a)}}}\right] d z
$$

Similar as in [8], we have

$$
\begin{gathered}
E\left[\frac{e^{\frac{-z \psi^{2}}{T} \int_{0}^{T} \frac{d s}{X_{s}}}}{X_{T}^{\frac{1}{2(1-a)}}}\right]=\frac{1}{2^{\nu} x^{m}} e^{-\frac{2 k x}{\sigma^{2}\left(e^{k T}-1\right)}+k m T}\left(\frac{2 k e^{k T}}{\left(e^{k T}-1\right) \sigma^{2}}\right)^{-m+\frac{1}{2(1-a)}-\frac{\nu}{2}} \\
\times \quad\left(\frac{4 k^{2} x}{\sigma^{4} \sinh ^{2}\left(\frac{k T}{2}\right)}\right)^{\nu / 2} \frac{\Gamma\left(1+m-\frac{1}{2(1-a)}+\frac{\nu}{2}\right)}{\Gamma(1+\nu)}{ }_{1} F_{1}\left(\beta, 1+\nu, \frac{2 k x}{\sigma^{2}\left(e^{k T}-1\right)}\right),
\end{gathered}
$$


where $\mu=\frac{z \psi^{2}}{T}$. Therefore, the value of a put option on variance at time zero is

$$
\begin{aligned}
P_{v}\left(0, S_{0}^{\delta_{*}}\right) & =X_{0}^{\frac{1}{2(1-a)}} E\left[\left(\frac{K}{X_{T}^{\frac{1}{2(1-a)}}}-\frac{\frac{\psi^{2}}{T} \int_{0}^{T} \frac{d s}{X_{s}}}{X_{T}^{\frac{1}{2(1-a)}}}\right)^{+}\right] \\
& =\frac{x^{\frac{1}{2(1-a)}}}{2 \pi i} \int_{d-\infty i}^{d+\infty i} \frac{e^{z K}}{z^{2}}\left[\frac{1}{2^{\nu} x^{m}} e^{-\frac{2 k x}{\sigma^{2}\left(e^{k T}-1\right)}+k m T}\left(\frac{2 k e^{k T}}{\left(e^{k T}-1\right) \sigma^{2}}\right)^{-m+\frac{1}{2(1-a)}-\frac{\nu}{2}}\right. \\
& \left.\times\left(\frac{4 k^{2} x}{\sigma^{4} \sinh ^{2}\left(\frac{k T}{2}\right)}\right)^{\nu / 2} \frac{\Gamma\left(1+m-\frac{1}{2(1-a)}+\frac{\nu}{2}\right)}{\Gamma(1+\nu)} F_{1}\left(\beta, 1+\nu, \frac{2 k x}{\sigma^{2}\left(e^{k T}-1\right)}\right)\right] d z .
\end{aligned}
$$

The corresponding formula for a call option on variance can be obtained by using put-call parity.

To give an example, assume the parameters $x=1, T=1, a=\frac{2}{3}, \beta=\frac{\nu}{2}+\frac{1}{4}, k=0.052$, $\psi=\frac{3}{2}, m=\frac{3}{4}$ and $\nu=2 \sqrt{\frac{9}{16}+\frac{9 z}{2}}$; see [10],[8].

Table 5.1 displays the prices of put options on variance for various strike prices.

Table 5.1: Prices of put options on variance

\begin{tabular}{|c|c|}
\hline Strike Prices & Prices of put Options on variance \\
\hline 1 & $7.606 \times 10^{-4}$ \\
\hline 1.5 & 0.0223 \\
\hline 2 & 0.1013 \\
\hline 2.5 & 0.2428 \\
\hline 3 & 0.4345 \\
\hline 3.5 & 0.6632 \\
\hline 4 & 0.9191 \\
\hline
\end{tabular}

\section{Volatility Swaps}

Since there are no closed-form formulas for the price of volatility swaps, we will use a quasi-Monte Carlo simulation in the sequel. For details of quasi-Monte Carlo methods of this kind, we refer to [3]. 
The joint Laplace transform of $X_{T}$ and $\int_{0}^{T} \frac{d s}{X_{s}}$ is given by

$$
\begin{aligned}
& E\left(e^{-\lambda X_{T}-\mu \int_{0}^{T} \frac{d s}{X_{s}}}\right) \\
& =\frac{\Gamma(m+\nu / 2+1)}{\Gamma(\nu+1)} \frac{k \sqrt{x}}{\sigma^{2} \sinh (k T / 2)} x^{-\left(m+\frac{1}{2}\right)} \exp \left(\frac{k}{\sigma^{2}}\left(k \vartheta T+x-\frac{x}{\tanh (k T / 2)}\right)\right) \\
& \quad \times \frac{\sigma^{2} \sinh (k T / 2)}{k \sqrt{x} \hat{\alpha}^{\left(m+\frac{1}{2}\right)}} e^{\frac{k^{2} x}{2 \sigma^{4} \sinh ^{2}(k T / 2) \hat{\alpha}}} M_{-\left(m+\frac{1}{2}\right), \nu / 2}\left(\frac{k^{2} x}{\sigma^{4} \sinh ^{2}(k T / 2) \hat{\alpha}}\right),
\end{aligned}
$$

where $\hat{\alpha}=\frac{k}{\sigma^{2}}\left(1+\operatorname{coth}\left(\frac{k T}{2}\right)\right)+\lambda$ and $M_{s, r}(z)$ is the Whittaker function of the first kind, and in [10] the inverse Laplace transform with respect to $\lambda$ is explicitly given by

$$
\begin{aligned}
p(T, x, y)= & \frac{k}{\sigma^{2} \sinh (k T / 2)}\left(\frac{y}{x}\right)^{\left(\frac{k \vartheta}{\sigma^{2}}-\frac{1}{2}\right)} \\
& \exp \left(\frac{k}{\sigma^{2}}\left(k \vartheta T+x-y-\frac{x+y}{\tanh (k T / 2)}\right)\right) I_{\nu}\left(\frac{2 k \sqrt{x y}}{\sigma^{2} \sinh (k T / 2)}\right) .
\end{aligned}
$$

Here $I_{\nu}$ is the modified Bessel function of the first kind. Hence, to obtain the joint density of $\left(X_{T}, \int_{0}^{T} \frac{d s}{X_{s}}\right)$, we only need to invert a one-dimensional Laplace transform, which can be achieved via the Euler method from [1].

As shown in [3], the joint density $f(x, z)$ obtained by numerically inverting the Laplace transform can be mapped into the unit square by setting the exponential transforms, $x_{1}=1-\exp \left(-\lambda_{1} x\right), x_{2}=1-\exp \left(-\lambda_{2} z\right), x, z \in \Re^{+}$, and hence $x=\Psi_{1}^{-1}(x)=\frac{\log \left(1-x_{1}\right)}{-\lambda_{1}}$, $z=\Psi_{2}^{-1}(z)=\frac{\log \left(1-x_{2}\right)}{-\lambda_{2}}, \psi_{1}(x)=\lambda_{1} \exp \left(-\lambda_{1} x\right), \psi_{2}(z)=\lambda_{2} \exp \left(-\lambda_{2} z\right)$. For a given 
transformation $\left(\Psi_{1}, \Psi_{2}\right)$, the following formula is adopted from [3]:

$$
\begin{aligned}
& E\left(\frac{H\left(\int_{0}^{T} \frac{d s}{X_{s}}\right)}{X_{T}^{\frac{1}{2(1-a)}}}\right) \\
= & \int_{0}^{\infty} \int_{0}^{\infty} \frac{H(z)}{y^{\frac{1}{2(1-a)}}} f(y, z) d y d z \\
= & \int_{0}^{1} \int_{0}^{1} \frac{H\left(\Psi_{2}^{-1}\left(x_{2}\right)\right)}{\left(\Psi_{1}^{-1}\left(x_{1}\right)\right)^{\frac{1}{2(1-a)}}} f\left(\Psi_{1}^{-1}\left(x_{1}\right), \Psi_{2}^{-1}\left(x_{2}\right)\right) \prod_{j=1}^{2} \frac{1}{\psi\left(\Psi_{j}^{-1}\left(x_{j}\right)\right)} d x_{j} \\
= & \int_{0}^{1} \int_{0}^{1} \frac{H\left(\Psi_{2}^{-1}\left(x_{2}\right)\right)}{\left(\Psi_{1}^{-1}\left(x_{1}\right)\right)^{\frac{1}{2(1-a)}}} f\left(\Psi_{1}^{-1}\left(x_{1}\right), \Psi_{2}^{-1}\left(x_{2}\right)\right) \prod_{j=1}^{2} \frac{1}{\psi_{j}\left(\Psi_{j}^{-1}\left(x_{j}\right)\right)} d x_{j} \\
\approx & \frac{1}{N} \sum_{i=1}^{N} \frac{H\left(\Psi_{2}^{-1}\left(x_{i, 2}\right)\right)}{\left(\Psi_{1}^{-1}\left(x_{i, 1}\right)\right)^{\frac{1}{2(1-a)}}} f\left(\Psi_{1}^{-1}\left(x_{i, 1}\right), \Psi_{2}^{-1}\left(x_{i, 2}\right)\right) \prod_{j=1}^{2} \frac{1}{\psi_{j}\left(\Psi_{j}^{-1}\left(x_{i, j}\right)\right)},
\end{aligned}
$$

where $\left\{\left(x_{i, 1}, x_{i, 2}\right)\right\}_{i=1}^{N}$ is a two-dimensional quasi-Monte Carlo point set.

Recall that the price $V_{s}\left(t, S_{t}^{\delta_{*}}\right)$ of a volatility swap at time $t=0$ is:

$$
V_{s}\left(0, S_{0}^{\delta_{*}}\right)=\frac{S_{0}^{\delta_{*}} L \psi}{\sqrt{T}} E\left[\frac{\sqrt{\int_{0}^{T} \frac{1}{X_{s}} d s}}{X_{T}^{\frac{1}{2(1-a)}}}\right]-S_{0}^{\delta_{*}} L K_{s} B_{T}\left(0, S_{0}^{\delta_{*}}\right),
$$

where the expectation $E\left[\frac{\sqrt{\int_{0}^{T} \frac{1}{X_{s}} d s}}{X_{T}^{\frac{1}{2(1-a)}}}\right]$ is computed by using (6.1) with $H()=.\sqrt{\int_{0}^{T} \frac{1}{X_{s}} d s}$. 
Examples of volatility swaps are shown below in Table 6.1 with the same parameter setting as in Section 5. Table 6.1 displays numerical results for volatility swaps.

\begin{tabular}{|c|c|}
\hline maturity(years) & $\begin{array}{c}\text { volatility swaps } \\
\left(\lambda_{1}=0.5, \lambda_{2}=0.18\right)\end{array}$ \\
\hline $1 / 6$ & 0.5618 \\
\hline 0.25 & 0.6842 \\
\hline 0.5 & 0.79 \\
\hline 1 & 0.6323 \\
\hline 1.5 & 0.4384 \\
\hline 2 & 0.3076 \\
\hline
\end{tabular}

Table 6.1: Numerical results for volatility swaps.

\section{References}

[1] J. Abate, W. Whitt, Numerical inversion of Laplace transforms of probability distributions, ORSA J. Comput.7(1995) 36-43.

[2] L. Andersen, J. Andreasen, Volatility skews and extensions of the Libor market model, Appl. Math. Fin.7(2000)1-32.

[3] J. Baldeaux, L. Chan, E. Platen, Quasi-Monte Carlo methods for derivatives on realised variance of an index under the benchmark approach, The ANZIAM J.52 (CTAC2010) 727-741.

[4] M. Britten-Jones, A. Neuberger, Option prices, implied price processes, and stochastic volatility, J. Fin. 55 (2000) 839-866.

[5] P. Carr, R. Lee, Volatility derivatives, Annu. Rev. Fin. Econ. 1(2009) 1-21. 
[6] P. Carr, L. Wu, A Tale of two indices, J. Deri.13(2006) 13-29.

[7] P. Carr, J. Sun, A new approach for option pricing under stochastic volatility, Rev. Deri. Res. 10(2007) 87-150.

[8] L. Chan, E. Platen, Pricing and hedging of long dated variance swaps under a $3 / 2$ volatility model, J. Comput. Appl. Math. 278(2015) 181-196.

[9] J. Cox, Notes on option pricing I: Constant elasticity of variance diffusions, Unpublished note, Standard University, Graduate School of Business (1975).

[10] M. Craddock, K.A. Lennox, The calculation of expectations for classes of diffusion processes by Lie symmetry methods, Ann. Appl. Prob. 19(2009) 127-157.

[11] D. Davydov, V. Linetsky, Pricing and hedging path-dependent options under the CEV process, Manag. Sci. 47(2001) 949-965.

[12] R.J. Elliott, T.K. Siu, L. Chan, Pricing volatility swaps under Heston's stochastic volatility model with regime switching, Appl. Math. Fin. 14(2007) 41-62.

[13] R.J. Elliott, L. Chan, T.K. Siu, Option valuation under a regime-switching constant elasticity of variance process, Appl. Math. Comput. 219(2013) 4434-4443.

[14] D. Heath, E. Platen, Consistent pricing and hedging for a modified constant elasticity of variance model, Quant. Fin. 2(2002) 459-467.

[15] M. Jeanblanc, M. Yor, M. Chesney, Mathematical Methods for Financial Markets, Springer, Berlin Heidelberg New York (2006).

[16] J. Kallsen, J. Muhle-Karbe, M. Voß, Pricing options on variance in affine stochastic volatility models, Math. Fin. 21(2011) 627-641.

[17] M. Keller-Ressel, J. Muhle-Karbe, Asymptotic and exact pricing of options on variance, Fin. Stoch. 17(2013) 107-133. 
[18] C.F. Lo, P.H. Yuen, C.H. Hui, Constant elasticity of variance option pricing model with time dependent parameters, Int. J. Theor. Appl. Fin. 4(2000) 661-674.

[19] S. Miller, E. Platen, Real-world pricing for a modified constant elasticity of variance model, Appl. Math. Fin. 17(2010) 147-175.

[20] E. Platen, D. Heath, A Benchmark Approach to Quantitative Finance, Springer Finance, Springer (2010). 